\title{
Dark energy in the environments of the Local Group, the M 81 group, and the CenA group: the normalized Hubble diagram
}

\author{
P. Teerikorpi ${ }^{1}$, A. D. Chernin ${ }^{1,2,3}$, I. D. Karachentsev ${ }^{4}$, and M. J. Valtonen ${ }^{1}$ \\ 1 Tuorla Observatory, University of Turku, 21500 Piikkiö, Finland \\ e-mail: pekkatee@utu.fi \\ 2 Sternberg Astronomical Institute, Moscow University, 119899 Moscow, Russia \\ 3 Division of Astronomy, University of Oulu, 90014, Finland \\ ${ }^{4}$ Special Astrophysical Observatory, Nizhnii Arkhys 369167, Russia
}

Received 22 October 2007 / Accepted 27 February 2008

\section{ABSTRACT}

\begin{abstract}
Context. Type Ia supernova observations on scales of thousands of Mpc show that the global expansion of the universe is accelerated by antigravity produced by the enigmatic dark energy contributing $3 / 4$ of the total energy of the universe.

Aims. Does antigravity act on small scales as well as large? As a continuation of our efforts to answer this crucial question we combine high accuracy observations of the galaxy flows around the Local Group and the nearby M 81 and CenA groups to observe the effect of the dark energy density on local scales of a few Mpc.

Methods. We use an analytical model to describe non-uniform static space-time regions around galaxy groups. In this context it is useful to present the Hubble flow in a normalized Hubble diagram $V / H_{\mathrm{v}} R_{\mathrm{v}}$ vs. $r / R_{\mathrm{v}}$, where the vacuum Hubble constant $H_{\mathrm{v}}$ depends only on the cosmological vacuum density and the zero-gravity distance $R_{\mathrm{v}}$ depends on the vacuum density and on the mass of the galaxy group. We have prepared the normalized Hubble diagrams for the LG, M 81 and CenA group environments for different values of the assumed vacuum energy density, using a total of about 150 galaxies, for almost all of which the distances have been measured by the HST.

Results. The normalized Hubble diagram, where we identify dynamically different regions, is in agreement with the standard vacuum density $\left(\Omega_{\mathrm{v}}=0.77 h_{70}^{-2}\right)$, the out-flow of galaxies clearly being controlled by the minimum energy condition imposed by the central mass plus the vacuum density. A high vacuum density $1.6 h_{70}^{-2}$ violates the minimum energy limit, while a low density $0.1 h_{70}^{-2}$ leaves the start of the Hubble flow around 1-2 Mpc with the slope close to the global value obscure. We also consider the subtle relation of the zero-gravity radius $R_{\mathrm{v}}$ to the zero-velocity distance $R_{0}$ appearing in the usual retarded expansion around a mass $M$ : in a vacuumdominated flat universe $R_{0} \approx 0.76 R_{\mathrm{v}}$.

Conclusions. The normalized Hubble diagram appears to be a good way to present and analyze physically different regions around mass clumps embedded in cosmological vacuum. The most natural interpretation of the diagram is that the local density of the dark energy is approximately equal to the density known from studies on global scales.
\end{abstract}

Key words. cosmology: dark matter - cosmology: cosmological parameters - galaxies: Local Group

\section{Introduction}

Of the main parameters of the Friedmann model, the Hubble constant and dark matter density may be studied observationally in the relatively local universe. Dark energy has been an exception. It was detected from the high-redshift Hubble diagram (Riess et al. 1998; Perlmutter et al. 1999), is consistent with the CMB anisotropy (Spergel et al. 2003, 2007), and often has been regarded as having no observable effects in the local galaxy universe.

\subsection{Local cosmology}

After the first results from distant supernovae, we proposed that the dark energy (or cosmological vacuum) might be detectable in the local $(<10 \mathrm{Mpc})$ Hubble flow where Edwin Hubble discovered his law (Chernin et al. 2000, Paper I; Chernin 2001; Baryshev et al. 2001). Our key argument was the fact that the vacuum antigravity dominates over the matter gravity of the Local Group at distances larger than $\approx 1.5 \mathrm{Mpc}$ from the centre of the group and its uniform density might provide the dynamical background for a regular quiescent local expansion.
The gravity force $\left(\propto 1 / r^{2}\right)$ of mass $M$ dominates the antigravity force $(\propto r)$ at distances $<R_{\mathrm{v}}$. At the distance

$R_{\mathrm{v}}=\left(G M A^{2}\right)^{1 / 3}=\left(\frac{3}{8 \pi} M / \rho_{\mathrm{v}}\right)^{1 / 3}$

gravity and antigravity balance each other, while beyond $R_{\mathrm{v}}$ antigravity dominates, and the acceleration is positive (Paper I). In this expression the parameter $A$ depends on the vacuum density $\rho_{\mathrm{v}}\left(A=\left(\frac{8 \pi G}{3} \rho_{\mathrm{v}}\right)^{-1 / 2}\right.$, corresponding to the universal vacuum Hubble constant $\left.H_{\mathrm{v}}=1 / A\right)$.

To observe the Hubble law requires a low-dispersion expansion flow. Indeed, realistic simulations of $10 \mathrm{Mpc}$ scale environments similar to that of the Local Group have shown that, in addition to a small matter density excess, dark energy is needed in order to produce the observed cool local flow, confirming our original idea (Governato et al. 1997; Macciò et al. 2005). In general, the gravitation-dominated regions have scales not far from the correlation length in the galaxy distribution, possibly also linking this number to the cosmological vacuum (Teerikorpi et al. 2005). 


\subsection{The Hubble law around galaxy groups}

The regular expansion flow in the local inhomogeneous galaxy universe has been seen as an enigma, most clearly by Sandage (1999). In fact, focusing on near neighbourhoods of galaxy groups, one may formulate the paradox on these megaparsec scales in terms of the Einstein \& Straus (1945) "vacuole" model: in the homogeneous Friedmann model one might carve out a sphere and replace it with a mass point of the same mass in the centre of the empty sphere, without globally affecting the model. Inside this vacuole there is a static space-time, even though the border of the sphere expands with the global expansion rate. The zero-gravity surface $R_{\mathrm{V}}$ is inside the vacuole (Chernin et al. 2006).

The matter comprising the central concentration must have originated in gravitational gathering of initially almost uniformly distributed and expanding primordial matter in a volume whose present size roughly corresponds to the ES vacuole (we denote its radius by $R_{\mathrm{ES}}$ ). During this non-linear process the regular Hubble expansion is necessarily brushed away and test particles in the vacuole follow trajectories in the static space-time. If we still see regular expansion in this volume, it must have originated elsewhere than in the primordial expansion.

As part of our answer to this enigma, computer simulations (Chernin et al. 2004, 2007d) have treated the Local Group as a binary (the Milky Way and M 31), as in the classical KahnWoltjer (1959) model, and describe the history of the local flow during the 12-13 Gyr since the formation of the group. They indicate that the Little Bang model (Valtonen et al. 1993, 1995) for the initial chaotic state of the Local Group (see also van den Bergh 2003) could produce a flow of galaxies across the zero-gravity surface. Their radial velocities are then amplified by the antigravity push of the cosmic vacuum outside the zerogravity surface.

In the present paper we combine the galaxy group data previously discussed separately for three groups (the Local Group, the M 81 group, the CenA group) by Chernin et al. (2007a-c) into one normalized Hubble diagram.

\section{The normalized Hubble diagram: the principle}

In order to see what happens to the test particles that have left the central region of the vacuole we consider them in the static, spherically symmetric space-time that is a well-known GR solution for a point-like gravitating body on a vacuum background. We have discussed this static space-time in Chernin et al. (2006). It contains the abovementioned constant parameters $M$ and $A$.

The particles inside the vacuole move along geodesics in this space-time. When deviations from the Newtonian space-time are small (as they are near small groups), the metric takes the form $\approx 1-G M / r-\frac{1}{2}(r / A)^{2}=1+U(r)$, where $U(r)$ is the gravitational potential of the Newtonian theory. In particular, the radial motions of the particles are practically the same as predicted by the Newtonian equation of motion, where the forces acting are the gravity of the central mass and the antigravity of the vacuum.

The total energy of each dwarf galaxy that has escaped from the gravity potential well of the galaxy group must exceed the maximal value of the potential $U$

$E>U_{\max }=-\frac{3}{2} G M / R_{\mathrm{v}}$

which is reached at the distance $R_{\mathrm{v}}$.

\subsection{Normalized distance and normalized velocity}

It is quite convenient to normalize our equations to the zerogravity distance $R_{\mathrm{v}}$ and consider the Hubble diagram with normalized distance and velocity ( $x$ - and $y$-axes): $x=r / R_{\mathrm{V}}$ and $y=V / H_{\mathrm{v}} R_{\mathrm{v}}=V / V_{\mathrm{v}}$ (the quantity $V_{\mathrm{v}}$ is formally the speed of the vacuum flow at the distance $R_{\mathrm{v}}$ ). Then radially moving test particles will move along curves, which depend only on the constant total mechanical energy $E$ of the particle:

$y=x\left(1+2 x^{-3}-2 \alpha x^{-2}\right)^{1 / 2}$.

Here $\alpha$ parameterizes the energy, so that $E=-\alpha G M / R_{\mathrm{v}}$. The present position of the particle on its own curve depends on the initial conditions (distance and time), but each curve has a velocity minimum at $x=1$ (i.e. $r=R_{\mathrm{V}}$ ).

The straight line $y=x$ gives the vacuum Hubble flow, which is asymptotically approached by the out-flying particles beyond $x=1$.

The energy with $\alpha=3 / 2$ is special: it is the minimum energy that still allows a particle initially below $x=1$ to reach this zerogravity border (and if the energy is slightly larger) to continue to the vacuum-dominated region $x>1$, where it starts accelerating. In the ideal case one does not expect particles with $x>1$ below this minimum velocity curve. If one changes the values of the parameters $M$ and $\rho_{\mathrm{v}}$ (or $A$ ), the $y-x$ curves do not change, but the normalized positions of observed test particles (dwarf galaxies) do change as $R_{\mathrm{v}}$ and $H_{\mathrm{v}}$ change. For example, if one lets the vacuum density decrease, the zero-gravity distance grows, and the normalized distance $x$ decreases for a galaxy with the fixed distance $r^{1}$.

Below we give two useful formulae connecting the vacuum Hubble constant $H_{\mathrm{v}}$ and the vacuum density $\rho_{\mathrm{v}}$ to the mass $M$ and the location of the zero-gravity border $R_{\mathrm{v}}$ :

$H_{\mathrm{v}}=60\left(\frac{M}{1.5 \times 10^{12} M_{\odot}}\right)^{1 / 2}\left(\frac{1.3 \mathrm{Mpc}}{R_{\mathrm{v}}}\right)^{3 / 2} \mathrm{~km} \mathrm{~s}^{-1} \mathrm{Mpc}^{-1}$

$\rho_{\mathrm{v}}=7 \times 10^{-30}\left(\frac{M}{1.5 \times 10^{12} M_{\odot}}\right)\left(\frac{1.3 \mathrm{Mpc}}{R_{\mathrm{v}}}\right)^{3} \mathrm{~g} / \mathrm{cm}^{3}$.

\subsection{Regions in the normalized Hubble diagram}

In Fig. 1 we show a schematic diagram on the different regions in the normalized Hubble diagram - it will be helpful when inspecting the diagrams based on real data.

Below $R=R_{\mathrm{v}}$, we have indicated the positive minimum velocity curve and its negative symmetric counterpart. It is between these curves where one expects galaxies which have had energies a little below the escape energy $-\frac{3}{2} G M / R_{\mathrm{v}}$, slowly moving outwards $(V>0)$ or falling back $(V<0)$ towards the group. This interior of this region may be regarded as defining the group as a dynamical entity: a galaxy here now will not escape beyond $R_{\mathrm{V}}$ unless it obtains in the future sufficient energy from an interaction.

At $R=R_{\mathrm{v}}$, the minimum velocity curve reaches zero after which it starts increasing. It defines the lower envelope for the

\footnotetext{
1 One should not confuse the present "normalized distance" with a similar term introduced by Teerikorpi $(1984,1997)$ and then used in many studies of the Hubble constant though the goals are similar - to reveal, by a suitable transformation of data, physically (here) or statistically (in Hubble constant studies) similar regions between different samples.
} 


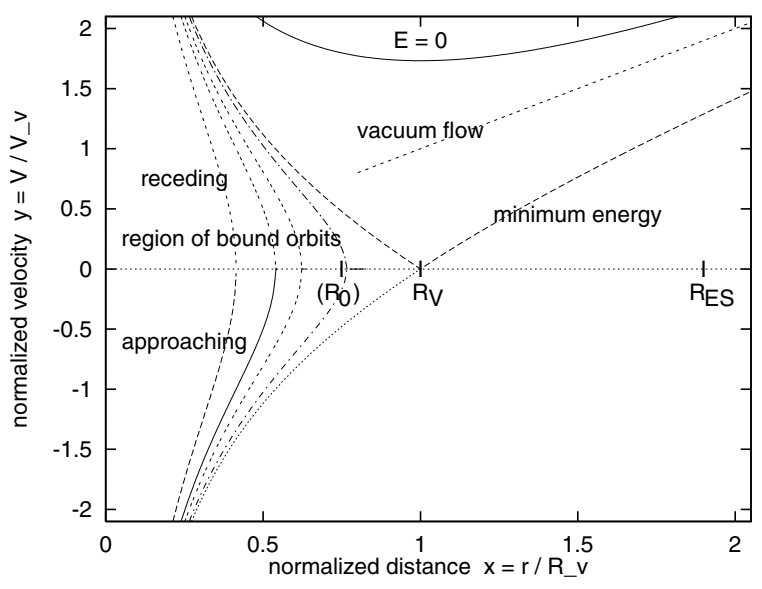

Fig. 1. A schematic diagram showing different regions in the normalized Hubble diagram around a mass concentration in the vacuum. In the region of bound orbits a dwarf galaxy cannot move into the region of the vacuum flow unless it receives extra energy as a result of an interaction with other galaxies.

permitted locations of out-flowing galaxies in the Hubble diagram. One also expects a - less sharp - upper envelope, represented by an iso-energy curve characterizing the highest-velocity tail of galaxies escaping from the group. The curve $E=0=\alpha$ is a convenient reference.

We have also indicated in Fig. 1 the interesting distances appearing in study of galaxy groups, $R_{0}, R_{\mathrm{v}}$ and $R_{\mathrm{ES}}$ (for the standard flat model; see for a discussion in Sect. 4.2).

\section{The Hubble diagram for the group environments}

We have previously (Chernin et al. 2007a) discussed the data for the dwarf galaxies around the Local Group. The distance data come mainly from high accuracy observations of the TRGB (Tip of the Red Giant Branch) using the Hubble Space Telescope as reported by Karachentsev and his team in a series of papers (references e.g. in Karachentsev et al. 2007). Here we combine those data with similar observations of the M 81 group (Chernin et al. 2007b). This group centered on the M 81-M 82 pair in Ursa Major is at the small distance of about $4 \mathrm{Mpc}$ and is about equally massive as the Local Group. Thus it is a main candidate for a comparative study. We give the distances and velocities refered to the center of the group. We also indicate the galaxies usually regarded as members of the groups (refered to by "-m", in the symbol lists of the diagrams).

\subsection{The Hubble diagram for the $L G$ and the $M 81$ group}

In the first approximation we use the same mass $1.5 \times 10^{12} M_{\odot}$, hence the same zero-gravity distance $R_{\mathrm{v}}=1.3 \mathrm{Mpc}$ for both the LG and the M 81 group. Figure 2 shows the normalized Hubble diagram for the environments of the two groups, with appropriate symbols. Here the vacuum density $\rho_{\mathrm{v}}=7 \times 10^{-30} \mathrm{~g} / \mathrm{cm}^{3}$ corresponds to $\Omega_{\mathrm{v}}=0.77 h_{70}^{-2}$ and $H_{\mathrm{v}}=60 \mathrm{~km} \mathrm{~s}^{-1} \mathrm{Mpc}^{-1}$ (and results in the units $R_{\mathrm{v}}=1.3 \mathrm{Mpc}$ and $H_{\mathrm{v}} R_{\mathrm{v}}=78 \mathrm{~km} \mathrm{~s}^{-1}$ for the $x$ - and $y$-axis, respectively). This "standard" vacuum density describes well the observed outflow around both groups, and the energy condition to overcome the potential well $(E>$ $\left.-\frac{3}{2} G M / R_{\mathrm{V}}\right)$ is not violated in the relevant range $x=r / R_{\mathrm{v}}>1$.

On the contrary, doubling the value of $\rho_{\mathrm{v}}$ would shift half of the galaxies below the energy limit curve, which is forbidden

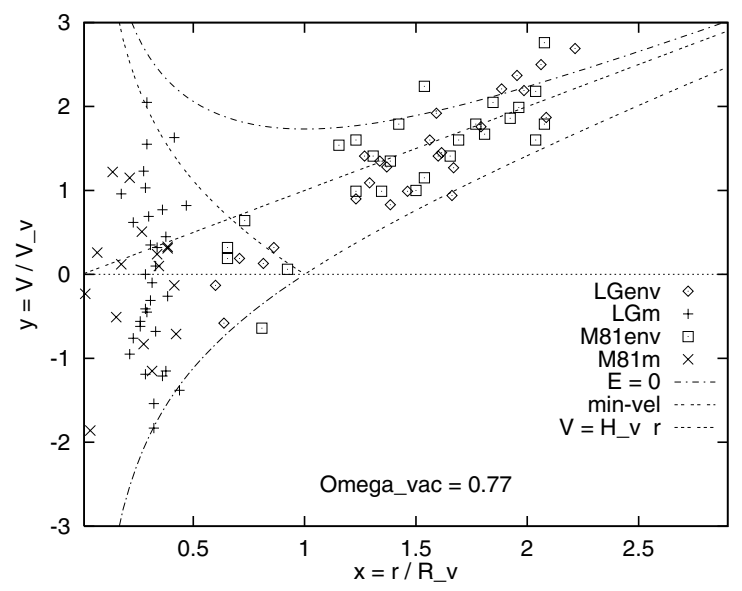

Fig. 2. The Hubble diagram for the environments of the LG and M 81 groups, for the "standard" vacuum density. The velocity-distance relation for the vacuum flow $y=x\left(V=H_{\mathrm{v}} r\right)$ is shown. The curve for the lower limit velocity $V_{0}$ is given below and above $x=1$; below $x=1$ its negative counterpart is shown.

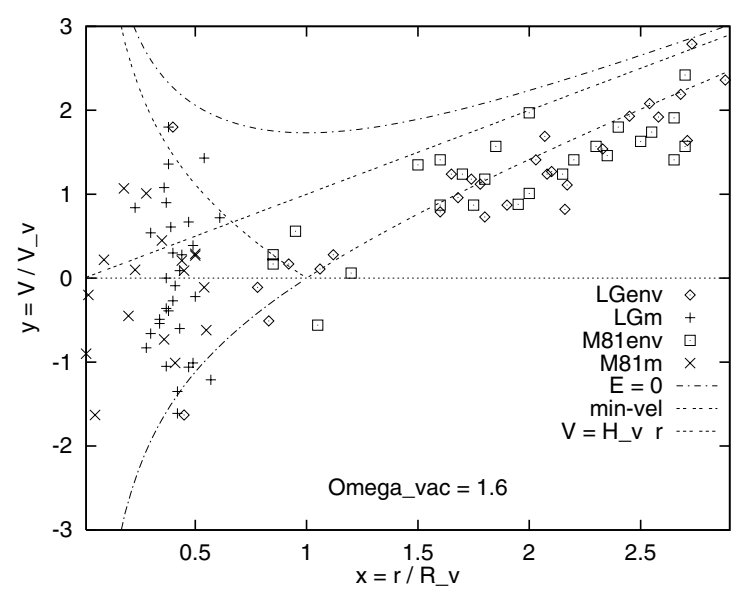

Fig. 3. The Hubble diagram for the LG and M 81 environments when the vacuum density is high $\left(R_{\mathrm{v}}=1.0 \mathrm{Mpc}, H_{\mathrm{v}} R_{\mathrm{v}}=88.9 \mathrm{~km} \mathrm{~s}^{-1}\right)$. Now many galaxies at $x>1$ violate the energy limit.

(see Fig. 3). On the other hand, if one lowers the vacuum density, say, to a twenty percent level then one loses any relation with the energy limit curves (Fig. 4).

\subsection{Adding the CenA group to the diagram}

The Centaurus A galaxy group is dominated by the giant elliptical Cen A. A less massive galaxy, the spiral M 83, is centered in another subconcentration. In this case the mass of the CenA concentration (around 6 to $8 \times 10^{12} M_{\odot}$; Karachentsev et al. 2007) is significantly larger than that of the Local Group or the M 81 Group and one must use a different normalization for CenA data, now corresponding to $R_{\mathrm{V}} \approx 2.2 \mathrm{Mpc}$. Using the same data as in Chernin et al. (2007c) we have added in Fig. 5 the CenA members and surrounding galaxies, with distances and velocities calculated relative to Centaurus A. We show the combined diagram for the "standard" vacuum density. 


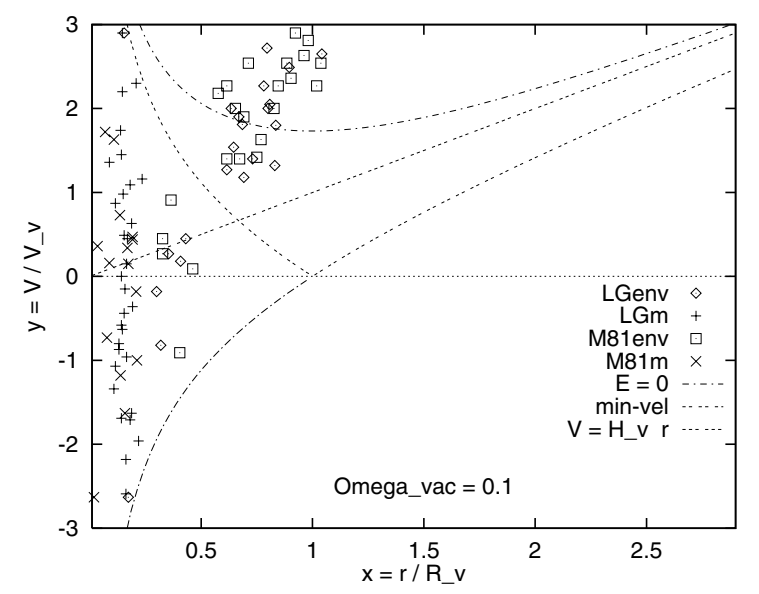

Fig. 4. The combined Hubble diagram for the environments of the LG and $\mathrm{M} 81$ groups, when the vacuum density is low, with the units $R_{\mathrm{v}}=$ $2.6 \mathrm{Mpc}$ and $H_{\mathrm{v}} R_{\mathrm{v}}=55.2 \mathrm{~km} \mathrm{~s}^{-1}$.

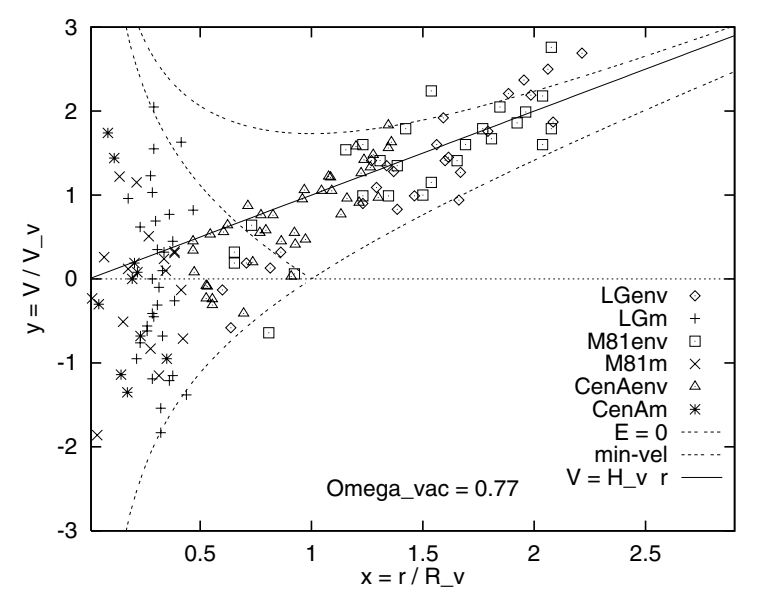

Fig. 5. The Hubble diagram for the environments of the LG, M 81, and CenA groups (the "standard" vacuum). The CenA data normalized to $R_{\mathrm{v}}=2.2 \mathrm{Mpc}$, due to the larger mass.

\section{Discussion}

Here we comment on the Hubble diagrams for different values of the vacuum density and discuss the important distances indicated in Fig. 1.

\subsection{The observed structure of the Hubble diagram}

In the normalized Hubble diagram constructed for the standard vacuum (Figs. 2, 5), the positive minimum energy curve and its negative velocity symmetric counterpart define the group(s) as a dynamical entity at $x<1$. It is just below $x=1$ where one expects a concentration of galaxies that have had energies a little below the escape energy, slowly moving outwards or falling back towards the group. The members of the "proper" group below $x \approx 0.5$ are confined between these curves.

Above $x=1$ the lower $\left(E=-\frac{3}{2} G M / R_{\mathrm{V}}\right)$ and upper envelopes $(E=0)$ define well the channel for out-flowing galaxies; in particular the galaxies lie above the minimum energy curve, following the vacuum Hubble flow $(y=x)$, which can be understood as due to the effective "cooling" of the flow in the dominating vacuum (Chernin et al. 2006).
The high vacuum density (Fig. 3) is excluded as violating the lower energy limit (like it is also rejected by Sne Ia data; cf. Fig. 8 in Riess et al. 2004).

For the weak vacuum $\left(\Omega_{\mathrm{v}}=0.1 h_{70}^{-2} ;\right.$ Fig. 4) the link with the vacuum flow $y=x$ and with other dynamical structures in Fig. 1 is lost. The Hubble flow occurs in the gravity-dominated region, perpendicular to the parabolic trajectory. As we note below, for $\Omega_{\mathrm{m}} \approx 0.2-0.25$ this area almost coincides with the E-S-region (Sect. 1.2), hence our model applies. Now the energy limit is not violated, but otherwise the distribution of energies (Fig. 4) is peculiar: a lack of galaxies within an interval around $\alpha=3 / 2$ plus a tail of many over-parabolic energies. Although some such scenario might be possible, we think that a smooth distribution of the total energies across $\alpha=3 / 2$, as in the standard vacuum case of Fig. 2, would be much more likely.

\subsection{The distances $R_{0}, R_{V}$ and $R_{E S}$ in the Hubble diagram}

In this model the interesting distances are $R_{\mathrm{V}}$ and the "vacuole" radius $R_{\mathrm{ES}}$ (see Fig. 1). Beyond $R_{\mathrm{ES}}$ begins the inter-group space, where the very local Hubble flow is more or less smoothly connected to the several Mpc scale flow. In our environment the latter flow is also affected by the dark energy (Paper I; Baryshev et al. 2001; Macciò et al. 2005; Teerikorpi et al. 2005), and the Hubble ratio there is not far from the very local one nor from the global one, all being linked to the vacuum constant $H_{\mathrm{v}}$.

The radius $R_{\mathrm{ES}}$ of the region from which the mass $M$ was gathered would be around $R_{\mathrm{ES}}=\left[M /(4 \pi / 3) \rho_{m}\right]^{1 / 3}=$ $\left(2 \rho_{\mathrm{v}} / \rho_{\mathrm{m}}\right)^{1 / 3} R_{\mathrm{v}} \approx 1.9 R_{\mathrm{v}}$ for the standard value $\rho_{\mathrm{v}} / \rho_{\mathrm{m}}=$ $0.77 / 0.23$ (for the ratio $0.8 / 0.2, R_{\mathrm{ES}}$ would be exactly $2 R_{\mathrm{V}}$ ). This general formula is valid not only for the flat model. For example, with $\rho_{\mathrm{v}} / \rho_{\mathrm{m}}=0.1 / 0.23$ one obtains $R_{\mathrm{ES}} \approx 0.95 R_{\mathrm{v}}$, which could apply to the case in Fig. 4.

How do these distances relate to the familiar zero-velocity radius $R_{0}$ expected in retarded expansion? This situation is generally treated with Tolman-Bondi solutions around a spherically symmetric density enhancement in the expanding background. The fact that $R_{0}$ is often used in mass determinations makes the above question relevant.

First, we emphasize that the "zero-gravity point" at $\left(r / R_{\mathrm{V}}=1, V=0\right)$ should not be viewed as the same thing as the classical zero-velocity distance $R_{0} . R_{\mathrm{v}}$ marks the position of the potential maximum within static space and depends only on the central mass $M$ and the vacuum density $\rho_{\mathrm{v}}$. $R_{0}$ depends on the mass $M$ (or on the density excess relative to the background density) and on the density parameter $\Omega_{\mathrm{m}}$ and, less strongly, on the vacuum density parameter $\Omega_{\mathrm{v}}$.

Secondly, thanks to the coupling between these two parameters in flat space, $\Omega_{\mathrm{m}}+\Omega_{\mathrm{v}}=1$, the numerical values of $R_{\mathrm{v}}$ and $R_{0}$ can be rather close to each other when $\Omega_{\mathrm{v}}$ dominates, as we show below.

The generalized expression for the zero-velocity (turn-over) radius $R_{0}$ around the mass $M$ (Lynden-Bell 1981; Sandage 1986) in the flat universe containing vacuum energy is (Karachentsev et al. 2007):

$R_{0}^{3}=M\left(8 G / \pi^{2}\right) H_{0}^{2} f\left(\Omega_{\mathrm{m}}\right)^{2}$

where

$f\left(\Omega_{\mathrm{m}}\right)=\Omega_{\mathrm{v}}^{-1}-\frac{1}{2} \Omega_{\mathrm{m}} \Omega_{\mathrm{v}}^{-\frac{3}{2}} \operatorname{arccosh}\left[\left(2 / \Omega_{\mathrm{m}}\right)-1\right]$.

Together with Eq. (1), one can derive

$\left(R_{0} / R_{\mathrm{v}}\right)^{3}=8(f / \pi)^{2} \Omega_{\mathrm{v}}$. 
For example, for $\Omega_{\mathrm{v}}=0.77$, one obtains $R_{0} / R_{\mathrm{v}}=0.76$, while for $\Omega_{\mathrm{v}}=0.95$, the result is $R_{0} / R_{\mathrm{v}}=0.88$. For small $\Omega_{\mathrm{v}}=0.1$ one obtains $R_{0} / R_{\mathrm{v}}=0.34$.

Thus we see that when the vacuum density dominates, as in the standard model, the zero-gravity distance is somewhat larger than the zero-velocity distance expected from ordinary retarded expansion. The three important distances $\left(R_{0}<R_{\mathrm{V}}<R_{\mathrm{ES}}\right)$ are roughly related as $R_{0} \approx 0.76 R_{\mathrm{v}} \approx 0.40 R_{\mathrm{ES}}$. Hence if the static space-time exists around a mass concentration as a result of the collapse (a total withdrawal from primordial expansion), the nearby Hubble flow does not show the classical turn-over, but the structure of the Hubble diagram is determined by the gravity - antigravity interplay in the non-Friedmann region.

There is a subtle implication for mass determination. One might interpret (incorrectly) this new kind of situation in terms of retarded expansion. Then if one takes the "zero-velocity distance" somewhat below the limit $R_{\mathrm{v}}$ (where slowly receding and approaching galaxies are concentrated; cf. Figs. 1 and 2), one happens to obtain approximately the correct mass, because $R_{0} \approx 0.76 R_{\mathrm{v}}$. In order to have an independent mass value from which to infer $R_{\mathrm{v}}$, we preferred in Chernin et al. (2006) to base the LG mass (as here: $1.5 \times 10^{12} M_{\odot}$ ) on individual measurements of the Milky Way and M 31, or to fix the total mass to the mass-to-luminosity ratio of the group.

There are systems still in the process of collapse (within a non-static space-time metric), showing a genuine zero-velocity surface dividing regions of retarded expansion and collapse. The Virgo supercluster is apparently such a case, the zero-velocity radius being observed to lie roughly midway between the Local Group and the Virgo cluster (Teerikorpi et al. 1992).

\section{Conclusions}

We summarize the main results of the present study:

- We introduce the concept of the combined normalized Hubble diagram in order to study the outflow of dwarf galaxies in the few Mpc environments of galaxy groups. The normalization is based on the zero-gravity distance $R_{\mathrm{v}}$ and on the vacuum Hubble constant $H_{\mathrm{v}}$, arising in the static space-time model describing the Einstein-Straus-like vacuole around a galaxy group.

- We have constructed the normalized Hubble diagram for the near environments of the LG, M 81, and CenA groups using the largest available collection of galaxies with distances mainly measured by the HST.

- The normalized Hubble diagram for the neighborhoods of these groups is consistent with the trajectories of galaxies flowing across the local zero-gravity borders, with the vacuum density $\rho_{\mathrm{v}}=7 \times 10^{-30} \mathrm{~g} / \mathrm{cm}^{3}$ (and $\Omega_{\mathrm{v}}=0.77 h_{70}^{-2}$ ). A value twice as high is excluded by the total energy criterion. With a value, say, five times less one would lose any relation with the expected dynamic structure in the Hubble diagram and, e.g., the start of the Hubble flow around 1-2 Mpc, in the now gravity-dominated E-S region, would remain obscure. We conclude that the local dark energy density is close to the globally measured $\rho_{\mathrm{v}}$, agreeing with our earlier estimate from the LG environment alone.

- For a fixed central mass, the zero-gravity distance $R_{\mathrm{v}}$ is about $30 \%$ larger than would be the zero-velocity radius $R_{0}$ in classical retarded expansion in a vacuum-dominated $\Omega=1$ world. One should be aware of how the situation described here differs from the retarded expansion, where one often uses the assumed $R_{0}$ for mass determination.

Acknowledgements. We thank the anonymous referee for useful remarks and suggestions. A.C. was partly supported by the RFBR grant 06-02-16366. I.K. was partly supported by the RFBR grant 07-02-00005 and the DFG-RFBR grant 06-02-04017.

\section{References}

Baryshev, Yu., Chernin, A., \& Teerikorpi, P. 2001, A\&A, 378, 729

Chernin, A. D. 2001, Physics-Uspekhi, 44, 1099

Chernin, A. D., Teerikorpi, P., \& Baryshev, Yu. V. 2000, Adv. Space Res., 31, 459 (Paper I)

Chernin, A. D., Karachentsev, I. D., Valtonen, M. J., et al. 2004, A\&A, 415, 19

Chernin, A. D., Teerikorpi, P., \& Baryshev, Yu. V. 2006, A\&A, 456, 13

Chernin, A. D., Karachentsev, I. D., Teerikorpi, P., et al. 2007a [arXiv: 0706.4068]

Chernin, A. D., Karachentsev, I. D., Kashibadze, O. G., et al. 2007b, Astrophys., 50,405

Chernin, A. D., Karachentsev, I. D., Makarov, D. I., et al. 2007c, A\&A Trans., 26,275

Chernin, A. D., Karachentsev, I. D., Valtonen, M. J., et al. 2007d, A\&A, 467, 933

Einstein, A., \& Straus, E. G. 1945, Rev. Mod. Phys., 17, 120

Governato, F., Moore, B., Cen, R., et al. 1997, New Astron., 2, 91

Kahn, F. D., \& Woltjer, L. 1959, ApJ, 130, 705

Karachentsev, I. D., Tully, R. B., Dolphin, A., et al. 2007, AJ, 133, 504

Lynden-Bell, D. 1981, Observatory, 101, 111

Macciò, A. V., Governato, F., \& Horellou, C. 2005, MNRAS, 359, 941

Perlmutter, S., Aldering, G., Goldhaber, G., et al. 1999, ApJ, 517, 565

Riess, A. G., Filippenko, A. V., Challis, P., et al. 1998, AJ, 116, 1009

Riess, A. G., Strolger, L.-G., Tonry, J., et al. 2004, ApJ, 607, 665

Sandage, A. 1986, ApJ, 307, 1

Sandage, A. 1999, ApJ, 527, 479

Spergel, D. N., Verde, L., Peiris, H. V., et al. 2003, ApJS, 148, 175

Spergel, D. N., Bean, R., Doré, O., et al. 2007, ApJS, 170, 377

Teerikorpi, P. 1984, A\&A, 141, 407

Teerikorpi, P. 1997, ARA\&A, 35, 101

Teerikorpi, P., Bottinelli, L., Gouguenheim, L., \& Paturel, G. 1992, A\&A, 260, 17

Teerikorpi, P., Chernin, A., \& Baryshev, Yu. 2005, A\&A, 440, 791

Valtonen, M. J., Byrd, G. G., McCall, M. L., \& Innanen, K. A. 1993, AJ, 105, 886

Valtonen, M. J., McCall, M. L., Innanen, K. A., Zheng, J. Q., \& Byrd, G. G. 1995, in Dark Matter, ed. S. S. Holt, \& C. L. Bennett, AIP Conf. Proc., 336, 450

Van den Bergh, S. 2003 [arXiv: astro-ph/0305042]

Wilkinson, M. I., \& Evans, N. W. 1999, MNRAS, 310, 645 